THE SUITE OF DETECTORS FOR RHIC

W. Willis

Brookhaven National Laboratory

November 1984 
THE SUITE OF DETECTORS FOR RHIC

1. The Multispectrometer Energy Flow Detector:

This device, Fig. 1, has moderately good energy resolution and excellent angular resolution for energy flow in high multiplicity events. The distance from the interaction point to the front face of the calorimeter is sufficient to maintain the good angular resolution aljowed by the granularity of the readout; in order to observe. Iocalizied excitations generated in the event, jets or "super jets" from plasma excitations. The depth of the calorimeter does not have to be very large, because the energy is carried largely by numerous moderate energy particles, and a few per cent of leakage has little effect. For similar reasons, the use of a calorimeter with compensation to give equal electron and hadron response may not be necessary. In the first phase, no separate detection of electromagnetic energy is provided. If dedicated experiments show that direct electromagnetic radiation is detectable at the level of gross energy flow, a separate, very thin, layer for its measurement can be introduced.

This device is well suited for accurate and sensitive measurements of the $E_{T}$ spectrum in different rapidity regions, and a search for localized structures in energy flow produced with very low cross-sections. The emphasis is on large energy deposits, and the design can take advantage of this fact in the read out scheme to produce an economical and compact device.

The second role of this instrument is to provide a facility for a number of independent experiments of the detailed properties of the high energy density events by observing particles through small apertures, called here "ports", provided in the calorimeter. The calorimeter then 
selects events with large or specially configured energy flow and gives a eomplete map of the flow over all angles, while the several Instrumented ports provide detailed measurements on individual particles. This approach should not be too quickly identified with the measurement of inclusive spectra by small angle spectrometers familiar from hadron machines, because the particle multiplicities at RHIC are so very high indeed that a smaII aperture, that is one which is small enough not to distort the measurement of energy flow, still transmits a large number of particles, so that individual events may be statistically characterized. by features of individual particle spectra or quantum numbers.

Also, the port spectrometers will no doubt be designed with an emphasis on multiple particle correlations. Meanwhile, the number of particles in the spectrometers is kept to numbers less than 50 or 100 , which can be conveniently handled by conventional tracking and aata analysis techniques.

The calorimeter should be designed so that the ports can be of configurations adapted to different purposes. For example, "one dimensional". slits with large aspect ratio are particularly powerful for tracking in high density environments. Application can be foreseen for slits covering a range of either polar or azimuthal angles." For correlation studies, deviation of the relative particle angles along aIl directions is desired, and this can be achieved by " $\theta$ " and " $\phi$ " slits which intersect in the form of a cross, a kind of aperture synthesis. A port of square aperture is more damaging from the viewpoint of energy flow distortion and presents a more difficult tracking problem, but may be required for some purposes. It may be necessary to terminate it with another section of calorimeter to retain the energy flow accuracy.

A reasonable complement of ports in the one calorimeter might be something like the following:

$$
\begin{aligned}
& 2-\Delta \phi=0.2^{\circ}, 2^{\circ}<\theta<8^{\circ} ; \\
& 2-\Delta \phi=0.5^{\circ}, 8^{\circ}<\theta<20^{\circ} ; \\
& 1-\Delta \phi=20^{\circ}, \theta=30 \pm 0.5^{\circ} ; \\
& 2-\operatorname{cross} 45^{\circ}<\theta<135^{\circ}, \Delta \phi=2^{\circ} \theta \Delta \theta=2^{\circ}, \Delta \phi=90^{\circ} ; \\
& 1 \quad \Delta \phi=10^{\circ}, 85<\theta<95^{\circ} ; \\
& 1 \quad \Delta \phi=1^{\circ}, 20<\theta<160^{\circ} ;
\end{aligned}
$$


In Fig. 1, the ports have all, except the crosses, been shown in the plane of the cross-section, giving a misleading impression. In fact, $95 \%$ of the solid angle is covered by calorimeter.

It is evident that these nine ports should be available in principle to up to nine different experimental groups, but it may not be wise to commit them too heavily, at least for long periods, so that flexibility will be available to follow up new ideas or discoveries.

The types of studies suftable for the ports include:

- Spectra of identified particles, correlated with anguiar features in energy flow;

- n-body correlation of identical charged particles;

- photon spectra and search for direct photons;

- nuclear and anti-nuclear states;

- search for new particles,

- direct lepton production.

One can suppose that the port spectrometers range in scale from quite a small effort to that of a medium sized experiment. A weil-thought out plan for data acquisition is necessary in order to accommodate such a program efficiently.

\section{Muon problem}

The problem of $\mu^{+} \mu^{-}$measurement in high $\dot{E}_{T}$ events at RHIC is rather different from that at other collider experiments. The main region of interest is for $\mathrm{m}_{\mathrm{T}}=\left(\mathrm{m}_{\mu \mu}^{2}+\mathrm{p}_{\mathrm{T}}^{2}\right)^{1 / 2}$ a $1-20 \mathrm{GeV}$, though $Z^{\circ}$ production should also be studied as the "poor man's Drell Yan" giving a point at large mass. The precision required on the muon momenta is not very great particularly for large $P_{I}$ muons. On the other hand, the background from the decays of the large number of pions threatens to be disastrous. The main criterion for the design of this experiment will be rapid absorbtion of the mesons. This will involve special beam pipes, high density absorbers starting immediately, providing calorimetric energy flow information with the least possible reduction in density and angular resolution provided mainly by the shower profile in the absorber material, 
constraints lead to energy flow accuracy inferior to that in the normai calorimeter, but sufficient for selecting high $E_{T}$ events in correlation with the muon pairs.

The muon momentum measurement is performed outside the compact first absorber-calorimeter. The high precision chambers are interspersed in an iron structure providing a toroidal field. This detector-iron array must provide sufficient openings to allow alignment, which is possible because the first absorber provides most of the rejection against hadron punch through.

The desired rapidity coverage should extend from the fragmentation region, lab. angles of a few degrees, to the central region. It is reasonable, from the point of view of rates, to instrument only one side in the first phase.

3. Photon-Photon Collision Equipment

The RHIC operating with $137 \mathrm{Au}$ beams at a Iuminosity of $10^{26} \mathrm{~cm}^{-2} \mathrm{~s}^{-2}$ - will deliver about $10^{3}$ times the photon-photon collision rate obtained at PETRA or PEP, and a comparison of event tagging efficiencies can bring the factor to $10^{\circ}$. This can allow a dramatic advance in studies of QCD in particularly clean reactions. The had̆ronic background can be suppressed by selecting events in which the photons are emitted coherently and the nucleus remains intact in the beam pipe, while the mesons from the photon-photon collision appear in the central region.

A new field of study will be events where three or more photons have collided, giving a mass of the mesonic system beyond the cutoff for the two photon reactions, and giving $C=-1$ systems instead of $c=+1$.

The detector required is an adaptation of an existing conventional colliding beam detector for $\gamma=10 . \mathrm{GeV}$. The same detector can be used to measure mesonic systems created by the double Pomeron mechanism in pP of a $\alpha$ collisions. The mass spectrum is known to be completely different from that in photon-photon systems, and is probably dominated by gluon states. Comparison with the $q q$ and $q q \bar{q} \bar{q}$ dominated $r y$ and $r y r$ reactions should help in untangling the spectroscopy of exotic states. 


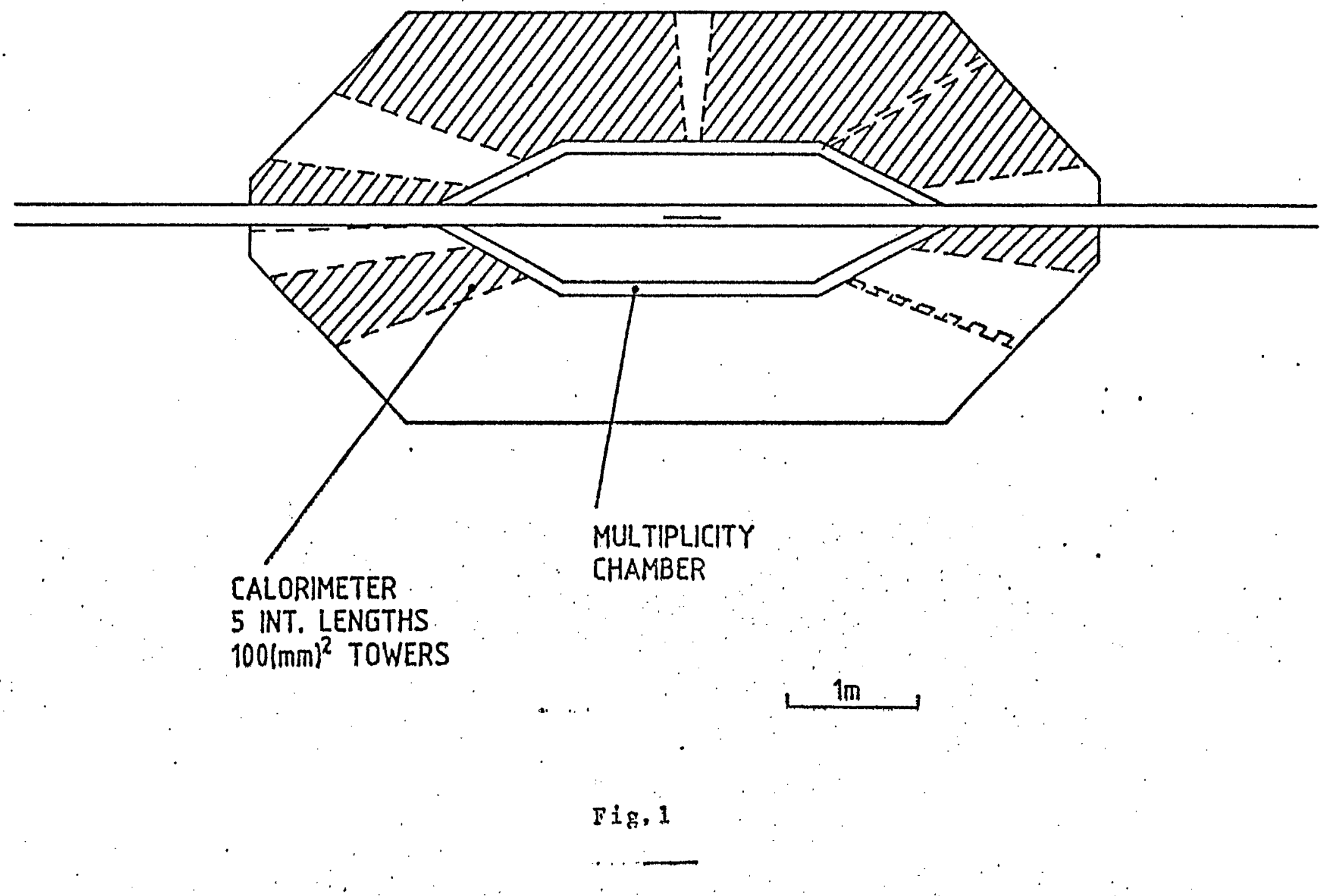




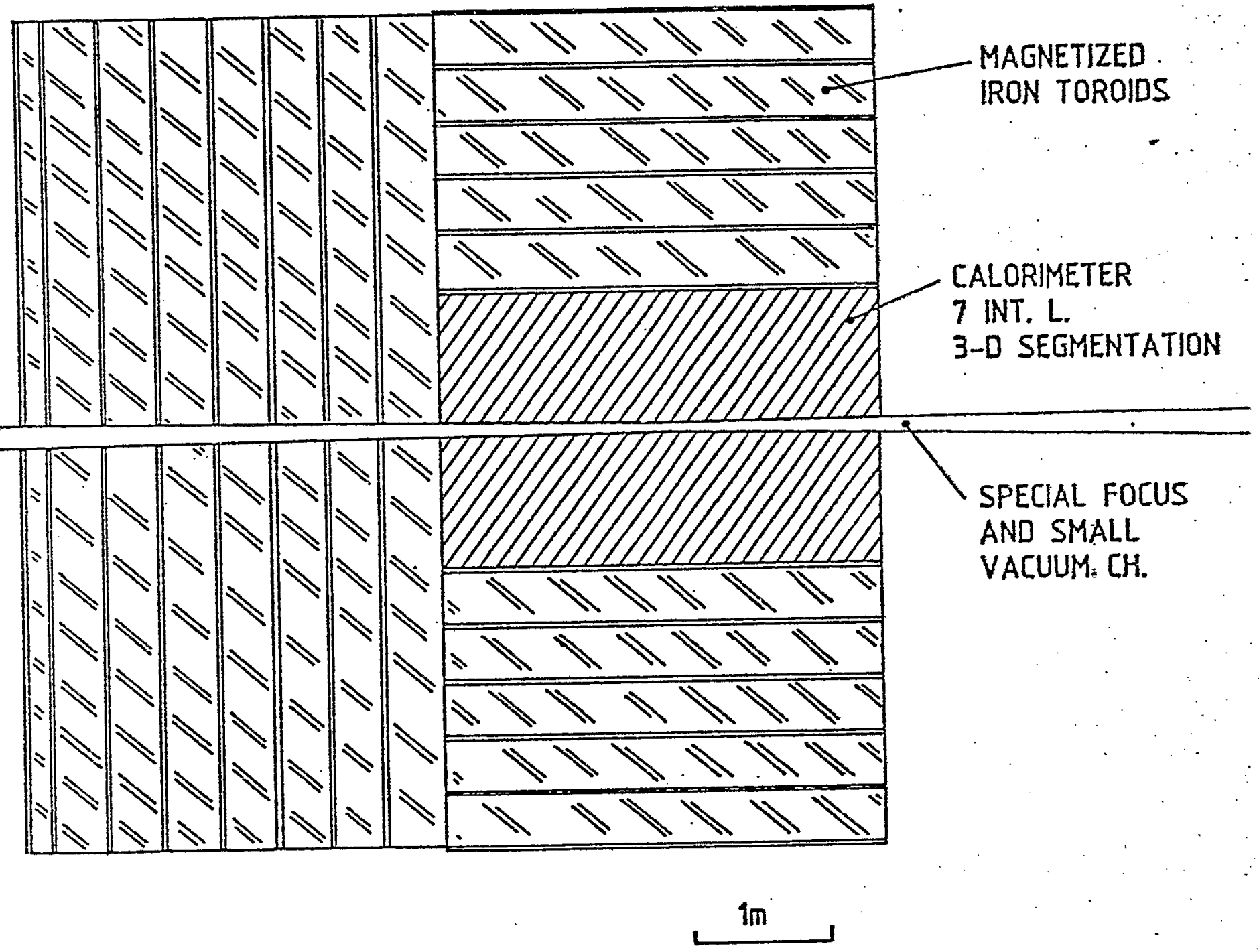

Fig. 2 ) 